\title{
Cardiac Graft from Donors with Extracorporeal Support-A Report of Two Cases
}

\author{
Bernd Panholzer ${ }^{1}$ Katharina Huenges ${ }^{1}$ Jochen Cremer $^{1}$ Assad Haneya ${ }^{1}$ \\ ${ }^{1}$ Department of Cardiovascular Surgery, University of \\ Schleswig-Holstein Campus Kiel, Kiel, Germany \\ Thorac Cardiovasc Surg Rep 2018;7:e33-e34.

\begin{abstract}
Address for correspondence Katharina Huenges, MD, Department of Cardiovascular Surgery, University of Schleswig-Holstein Campus Kiel, Arnold-Heller-Straße 3, Hs 18, Kiel 24105, Germany
\end{abstract} \\ (e-mail: Katharina.Huenges@uksh.de).
}

\begin{abstract}
Keywords

- extracorporeal support

- heart transplantation

- organ donors

The persistent global shortage of organ donors is still a major limitation for transplantation. Experiences of heart transplantation from donors with extracorporeal support are rare. Here, we report from two cases of donors who were supported by extracorporeal membrane oxygenation due to acute circulatory failure. In both cases, the direct postoperative course was uneventful and free from major complications. The patients were discharged to a rehabilitation clinic. Our experience suggests that the use of heart organ from carefully selected donors with extracorporeal support is possible and may lead to an excellent outcome.
\end{abstract}

\section{Introduction}

Although mortality rates on the waiting list have improved due to modern medical heart failure therapy and ventricular assist devices, heart transplantation (HTx) remains the best option for patients with end-stage heart failure. ${ }^{1}$ The persistent global shortage of organ donors is still a major limitation for transplantation. Previous studies reported successful outcomes of liver and kidney grafts procured from donors on extracorporeal membrane oxygenation (ECMO)., ${ }^{2,3}$ In contrast, experiences of HTx from donors with extracorporeal support are rare. Here, we report from two cases of donors who were supported by ECMO.

\section{Case Description}

\section{Case 1}

Granulomatosis with polyangiitis was diagnosed in a 20year-old male patient. Due to extended lung bleeding and isolated pulmonary failure, a veno-venous ECMO (CARDIOHELP, MAQUET Getinge Group, Rastatt, Germany) was implanted. Immediately after implantation, a significant improvement in gas exchange could be achieved, which remained stable over the following days. Unfortunately, a fatal subarachnoid bleeding occurred under ECMO support. After brain death assessment and complete clinical exam- ination were performed according to the guidelines, the patient was accepted as organ donor. Echocardiography was inconspicuous; it revealed a good heart function under very low-inotropic support. After evaluation, the donor heart was accepted for transplantation for a 51-year-old male patient with end-stage heart failure due to dilated cardiomyopathy. The cold ischemic time was 193 minutes. Postoperatively, a stable sinus rhythm was achieved and echocardiography showed an excellent biventricular function. The patient could be extubated 6 hours after surgery. The postoperative course was inconspicuous and free from major complications. With stable levels of immunosuppression therapy, stable cardiopulmonary function, and in absence of infection, the patient was discharged to a rehabilitation clinic 3 weeks after transplantation.

\section{Case 2}

A 35-year-old healthy woman had a witnessed drowning in a sea. The water temperature was $18^{\circ} \mathrm{C}$ and the submersion time was 30 minutes. On arrival of the emergency physician, the patient was unconscious, not breathing and the initial heart rhythm was asystolic. She was transferred to our institution, under continuous cardiopulmonary resuscitation (CPR) with the Lund University Cardiac Arrest System (LUCAS, Jolife AB, Lund, Sweden). On arrival, and after received

December 20, 2017

accepted after revision

February 23, 2018
DOI https://doi.org/

10.1055/s-0038-1661410. ISSN 2194-7635. (c) 2018 Georg Thieme Verlag KG
Stuttgart · New York

License terms

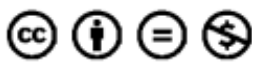


70 minutes of advanced life support without successful return of spontaneous circulation, a percutaneous venoarterial (femoro-femoral) ECMO (CARDIOHELP, MAQUET Getinge Group) was implanted during ongoing CPR. During the first 24 hours on ECMO, she regained an effective sinus rhythm and echocardiography showed a normal biventricular function. Three days later, the clinical diagnosis of brain death following anoxic brain injury was established. Echocardiographic examination showed a normal biventricular function without any inotropic support under an ECMO flow of $1.5 \mathrm{~L} / \mathrm{min}$. The HTx was performed in a 32-year-old female patient with a history of nonischemic cardiomyopathy being on left ventricular assist device (HVAD, HeartWare, Framingham, Massachusetts, United States). The intraoperative and postoperative courses were inconspicuous and free from major complications. The cold ischemic time was $182 \mathrm{~min}-$ utes and the patient was extubated 16 hours after surgery. The ventricular function was normal with a stable sinus rhythm. Three weeks after HTx, the patient could be discharged to a rehabilitation clinic.

\section{Discussion}

The persistent global shortage of donor organs limits the possibility of transplantation significantly. Paradoxically, not all suitable donor organs are used, and the question remains, how to increase the sources of potential organs available for transplantation. With increasing numbers of patients stabilized on ECLS as advanced life support in acute pulmonary as well cardiac failure-2,837 cases in Germany in 2016 ${ }^{4}$-these patients should be explored for possible organ donation in the situation of brain death. Patients with acute respiratory or circulatory failure, successfully stabilized with ECMO may be a so far not used option to increase the pool for donor organs. However, heart donation from potential donors on ECMO is complex due to concerns on postoperative graft failure and ethical issues. Yang et al reported that early extracorporeal support for salvaging brain-dead potential donors from cardiac death seems to be a practical strategy to increase the donor pool and preserve donor organs. ${ }^{5}$ Dalle Ave et al discussed the ethics associated with this approach and consented that ECMO should be considered only once brain death has been confirmed by standard brain death tests and once consent to organ donation has been documented or confirmed. $^{6}$

In this report, we described two successful cases of HTx with donors who were supported by ECMO due to acute circulatory failure. Immediately after ECMO implantation, cardiac function had significantly improved, as shown by clinical and echocardiographic assessment. In both cases, the recipient course and the heart function after transplantation were uneventful and free from major complications.

In conclusion, our experience suggests that the usage of heart from carefully selected donors with extracorporeal support is possible and is not inconsistent with an excellent outcome. Especially the situation Germany, with a persistent decrease in organ donation around one third compared with the data in 2012, these potential donors have to be carefully considered as potential heart donors in selected cases. A prospective analysis providing detailed hemodynamic, echocardiographic, as well clinical data will be necessary in a next step.

\section{References}

1 Colvin-Adams M, Smith JM, Heubner BM, et al. OPTN/SRTR 2013 Annual Data Report: heart. Am J Transplant 2015;15(Suppl 2):1-28

2 Carter T, Bodzin AS, Hirose H, et al. Outcome of organs procured from donors on extracorporeal membrane oxygenation support: an analysis of kidney and liver allograft data. Clin Transplant 2014;28(07):816-820

3 Hsieh CE, Lin HC, Tsui YC, et al. Extracorporeal membrane oxygenation support in potential organ donors for brain death determination. Transplant Proc 2011;43(07):2495-2498

4 Beckmann A, Funkat AK, Lewandowski J, et al. German Heart Surgery Report 2016: The Annual Updated Registry of the German Society for Thoracic and Cardiovascular Surgery. Thorac Cardiovasc Surg 2017;65(07):505-518

5 Yang HY, Lin CY, Tsai YT, Lee CY, Tsai CS. Experience of heart transplantation from hemodynamically unstable brain-dead donors with extracorporeal support. Clin Transplant 2012;26(05): 792-796

6 Dalle Ave AL, Gardiner D, Shaw DM. The ethics of extracorporeal membrane oxygenation in brain-dead potential organ donors. Transpl Int 2016;29(05):612-618 